\title{
Prostaglandin D2 Synthase Measurement
}

National Cancer Institute

\section{Source}

National Cancer Institute. Prostaglandin D2 Synthase Measurement. NCI Thesaurus.

Code C103432.

The determination of the amount of the prostaglandin D2 synthase in a sample. 